\title{
Comment on Dumoulin FL et al. Full-thickness resection with an over-the-scope device: possible translocation of adenoma tissue in a case of an incomplete resection at the appendix
}

In their recent publication, Dumoulin et al. report about a single-patient case of full-thickness resection of an adenoma at the base of the appendix with the FTRD (Full-Thickness Resection Device) device. Shortly after, the patient developed post-interventional appendicitis, and appendectomy was performed. Histologic evaluation of the appendix revealed a fragment of the colonic adenoma at the serosal side of the specimen. This finding leads the authors to reason that this tissue may have been translocated through the wall of the cecum or appendix by the endoscopic clip.

We consider this deliberation unlikely and want to contribute to the scientific discussion.

The FTRD clip, a modified OTSC clip, is not per se acting transmural. The teeth of the clip are not designed to cut through the wall of the intestinal tract. Since 2008, the original OTSC clip has been widely used for perforation closure, and experimental as well as clinical literature has shown that the closure is gas-tight. This underlines that the clip does not function transmurally. In rare situations, with very thin tissue employed, the tiny anchoring spikes at the teeth might reach the peritoneal level, as it is common with surgical staples. This has, however, never been reported as a clinical problem.

"Translocation of tissue," whatever this expression exactly means, has not been reported, not with the OTSC or the FTRD clip. The clip cannot "press" tissue through the wall of the colon, and it is not clear which speculative mechanism the authors are addressing here.

A loss of an adenoma tissue fragment would require an open colon wall defect to reach to the serosal side. This was apparently not the case. A "migration" of tissue through the intact wall is difficult to imagine.
What appears rather to be the likely cause for finding the tissue fragment in the histology specimen (in the fibrin) is an artifact resulting from sample preparation. For preparing the sample, it is required to remove the FTRD clip mechanically from the tissue. This is exerted by manually bending it open or by cutting it with tongs. Either way, this requires relevant mechanical force at the tissue level, resulting in laceration and opening of the colonic tissue at the clip anastomosis, which, at this point in time, shortly after the intervention, is not yet healed.

The authors should consider this aspect, which appears a quite probable cause for the histological finding they describe.

The authors speculate that the placement of the modified OTSC clip through neoplastic tissue in cases of incomplete resections poses a risk of tumor cell dissemination to the extra luminal side of the colon. In our opinion, it is not plausible that the FTRD clip would "translocate" tissue through the intact wall.

The resection through the lesion is a regular mechanism of action in piecemeal EMR (Endoscopic Mucosal Resection) and non-en-bloc ESD (Endoscopic Submucosa Dissection). Perforations with both techniques are not uncommon. This widely and long used practice has not led to clinical literature reports of tumor cell distribution.

Moreover, it does not seem helpful to draw such general conclusions from a single EFTR case, as presented by the authors.

Dumoulin has recently published a series of 182 colon ESD cases, 108 of which were in the right colon. Microperforation was reported in $9.3 \%$ of cases en bloc, and $\mathrm{R} 0$ resection rates were 88.4 and $62.6 \%$, respectively. Thus, in ESD a combination of colon perforation in the area of the adenoma and fragmentary and/or incomplete resection is apparently not unusual in regular clinical work. Even more so, we cannot see endoscopic fullthickness resection as being different in that regard.

It is obvious that resection through the target lesion is not intended in EFTR, but such as in other resection techniques, it may occasionally happen in clinical practice.

The $\mathrm{R} 0$ resection rate in the prospective, multicentric WallResect trial investigating full-thickness resection with FTRD in 181 patients was $76.9 \%$, which is higher than the $62.6 \%$ reported by Dumoulin for ESD. Most of the patients in WallResect had no further endoscopic options besides FTRD and may otherwise have undergone surgical resection.

While we agree with the authors that one should regularly avoid intentional cutting through neoplastic lesions, this may still occur during all endoscopic resection techniques, in piece-meal EMR, ESD, and EFTR, but not during EFTR in particular.

Data from WallResect show that, such as in ESD, R1 situations can normally be managed by subsequent endoscopic treatment with the staging of the lesion still permitting endoscopy or surgery in case of high risk stigmata.

\section{Competing interests}

Marc Schurr and Thomas Gottwald hold management positions with Ovesco Endoscopy AG. 
Bibliography

DOI http://dx.doi.org/10.1055/a-0655-7557

Endoscopy International Open 2018; 06: E1264E1265

(c) Georg Thieme Verlag KG

Stuttgart · New York

ISSN 2364-3722 resection at the appendix. Endosc Int Open 2018; 6: E622-E624

[2] Schmidt A, Beyna T, Schumacher T et al. Colonoscopic full-thickness resection using an over-the-scope device: a prospective multicentre study in various indications. Gut 2018; 67: 1280-1289

[3] Sauer M, Hildenbrand R, Oyama T et al. Endoscopic submucosal dissection for flat or sessile colorectal neoplasia > $20 \mathrm{~mm}$ : a European single-center series of 182 cases. Endosc Int Open 2016; 4: E895 - E900
[1] Dumoulin FL, Gorris DominikG, Berger S et al. Full-thickness resection with an overthe-scope device: possible translocation of
Prof. Dr. Med. Marc Schurr

Steinbeis University Berlin, $\mathrm{IHCl}$,

Dorfackerstr 26, 72074 Tuebingen, Germany

marc.schurr@ovesco.com 